\title{
INVESTIGANDO A CONJUNÇÃO ENTRE HISTÓRIA DA MATEMÁTICA E TECNOLOGIAS DE INFORMAÇÃO E COMUNICAÇÃO, POR MEIO DE UM LEVANTAMENTO BIBLIOGRÁFICO EM EVENTOS INTERNACIONAIS DE EDUCAÇÃO MATEMÁTICA
}

\author{
RESEARCHING THE CONJUNCTION BETWEEN THE HISTORY OF \\ MATHEMATICS AND INFORMATION AND COMMUNICATION \\ TECHNOLOGIES, BY A BIBLIOGRAPHIC SURVEY IN INTERNATIONAL \\ EVENTS OF MATHEMATICALEDUCATION
}

\author{
Giselle Costa de Sousa ${ }^{1}$ \\ Universidade Federal do Rio Grande do Norte \\ Allyson Emanuel Januário da Costa ${ }^{2}$ \\ Universidade Federal do Rio Grande do Norte
}

\begin{abstract}
Resumo
Este trabalho foi desenvolvido a partir de pesquisa qualitativa mediante um levantamento bibliográfico realizado em dois eventos internacionais, de Educação Matemática, a fim de identificar como se dá, ou se há, a conjunção entre a História da Matemática (HM) e as Tecnologias de Informação e Comunicação (TIC), através da catalogação dos trabalhos apresentados nestes eventos - VII Encontro Luso-Brasileiro de História da Matemática, no ano de 2014, em Óbidos, Portugal, e o VI Seminário Internacional de Pesquisa em Educação Matemática, 2015, em Pirenópolis, Estado de Goiás, Brasil - salientando como pesquisadores em Educação Matemática têm tratado esse tema. Consideramos que ocorre a conjunção proposta quando duas tendências em Educação Matemática, especialmente aqui tratamos do uso de História da Matemática apoiada por Tecnologias de Informação e Comunicação em prol do ensino de Matemática. A investigação desenvolvida nos possibilitou observar a importância de se continuar com esse levantamento e estudo, pois, embora haja constatações desta conjunção em alguns trabalhos, considera-se que uma ampliação deste estudo possa melhor delinear os parâmetros observados, dentre os quais destacamos: referencial; metodologia; tópicos de História da Matemática (tema histórico); recursos tecnológicos mais recorrentes; argumentos favoráveis para a conjunção (através de citações tiradas dos trabalhos encontrados) e ênfase educacional. Tendo em vista tais referenciais constatamos que o tema recorrente é Geometria com uso do recurso software Geogebra como mais frequente em pesquisas do tipo qualitativas, nem sempre com cunho educacional explícito. Essa pesquisa é vinculada ao projeto de pesquisa: Conexões Potenciais entre HM e TIC para o ensino de Matemática (PROPESQ/UFRN).
\end{abstract}

Palavras-chave: Levantamento; Conjunção; História da Matemática; TIC.

\footnotetext{
${ }^{1}$ gisellecsousa@ hotmail.com

2allysonecosta@gmail.com
} 


\begin{abstract}
This work was developed from qualitative research through a bibliographical survey done in two international events, of mathematics education, in order to identify how it is the conjunction between the History of Mathematics (HM) and Information and Communication Technologies (TIC) by cataloging the papers presented at these events - VII Luso-Brazilian Meeting of History of Mathematics, 2014, in Óbidos, Portugal, and the VI International Seminary on Research in Mathematical Education, 2015, in Pirenópolis, State of Goiás, Brazil - highlighting how researchers in mathematics education have understood this issue. We consider that the proposed conjunction occurs when two trends in Mathematics Education, especially here we deal with the use of History of Mathematics supported by Information and Communication Technologies for the teaching of Mathematics. The research developed enabled us to observe the importance to continue with this survey and study, because, although there are findings of this conjunction in some studies, it is considered that an extension of this study can better delineate the observed parameters, among which we highlight: referential; methodology; Topics of History of Mathematics (historical theme); Most recurrent technological resources; Favorable arguments for the conjunction (through quotes taken from the works found) and educational emphasis. In view of such references, we verified that the recurrent theme is Geometry with the use of the Geogebra software resource as more frequent in qualitative surveys, not always with an explicit educational character. This research is linked to the Research Project: Potential Connections between HM and TIC for teaching of Mathematics (PROPESQ/UFRN).
\end{abstract}

Keywords: Survey; Conjunction; History of Mathematics; TIC.

\title{
Introdução
}

O presente trabalho é vinculado à pesquisa: Conexões Potenciais entre História da Matemática (HM) e Tecnologias da Informação e Comunicação (TIC) para o ensino de Matemática (PROPESQ/UFRN), que propõe uma análise e apresenta possibilidades de aliança entre a HMe TIC para o ensino de Matemática de acordo com o processo de Investigação Matemática (IM) de Ponte, Brocado e Oliveira (2009), com os argumentos favoráveis ao uso de História na Educação Matemática de Miguel e Miorim (2008), bem como, as considerações de Borba (2007) acerca da Informática e Educação Matemática.

Alicerçado em tendências da Educação Matemática, particularmente: o uso da História da Matemática e o uso de recursos tecnológicos para o ensino da Matemática, investigamos indícios de como uma das tendências pode potencializar a outra, ou seja, se o uso da TIC pode fomentar a HM. Para tanto, realizamos um levantamento bibliográfico em dois anais de eventos, VII Encontro Luso-Brasileiro de História da Matemática e o VI SIPEM - Seminário Internacional de Pesquisa em Educação Matemática.

A escolha dos eventos se fez levando em consideração a importância para a área de Educação Matemática, o alcance dos trabalhos publicados/apresentados e a gama de pesquisadores e diversidade de pesquisas desenvolvidas, a história dos mesmos, bem 
como, o período, ou seja, a edição mais recente. Contudo, há também outros aspectos que justificam a escolha, dentre eles o acesso ao acervo digital, impresso ou on-line.

Para realização do levantamento, a respeito do tema já citado anteriormente, foram designadas algumas temáticas/subtemáticas para classificação dos trabalhos expostos. A primeira, Trabalhos de História da Matemática apoiado por TIC, é composta por trabalhos que tenham o uso de História da Matemática junto ao uso de softwares (1.1) ou outras mídias informáticas (1.2). Analogamente, na segunda, Trabalhos de TIC, estão os trabalhos que se utilizam das TIC, singularmente, no ensino de Matemática, seja pelo uso de softwares (1.1), seja pelo uso de quaisquer outras TIC (2.2). Na terceira temática, Trabalhos de História da Matemática, tratam-se de trabalhos que se utilizam da História da Matemática no Ensino de Matemática ou até mesmo problemas que possam ser, em um próximo estágio dessa pesquisa, resolvidos por meio de softwares. A quarta - e última temática - trata-se de qualquer outro trabalho de outras áreas da Educação Matemática dos demais trabalhos que não estão em outras categorias.

\section{Caminhos da Pesquisa}

Para a produção deste estudo foi utilizado o aspecto metodológico qualitativo, que caracteriza esse trabalho como uma pesquisa qualitativa do tipo bibliográfica que, conforme Neves (1996, p. 1), "tem por objetivo traduzir e expressar o sentido dos fenômenos do mundo social.”. Essa forma de pesquisa - qualitativa - está sendo bem utilizada entre os pesquisadores, pois oportuniza estudar o ser humano em suas relações sociais e os fenômenos que o cercam, nos mais diferentes ambientes (GODOY, 1995). Lima e Mioto (2007, p. 44) destacam que "Ao tratar da pesquisa bibliográfica, é importante destacar que ela é sempre realizada para fundamentar teoricamente o objeto de estudo, contribuindo com elementos que subsidiam a análise futura dos dados obtidos."

Para tanto, além da obtenção dos arquivos, os mesmos foram traduzidos, digitalizados e organizados em tabelas de modo a atender a categorização posterior cujos trabalhos da subtemática 1.1 foram analisados na íntegra, considerando alguns parâmetros já mencionados e detalhados a seguir. 


\section{Resultados}

a) Levantamento dos dados do VII Encontro Luso-Brasileiro de História da Matemática

É um evento que movimenta pesquisadores, em História da Matemática, brasileiros e portugueses, mas que também agrega pesquisadores de outras nacionalidades. Deste modo, há sempre oradores das duas nacionalidades cujas sociedades de HM são organizadoras. O VII LUSO ocorreu em Óbidos, Portugal, de 15 a 19 de outubro de 2014, mas o primeiro deles foi realizado em 1993, em Coimbra, Portugal.

A seguir, uma tabela que nos mostra, os dados obtidos a partir das comunicações apresentadas nesse evento, classificadas/categorizadas em nossas temáticas de pesquisa.

Tabela 1 - Comunicações apresentadas no VII Encontro Luso-Brasileiro de História da

Matemática, classificadas de acordo com as temáticas/subtemáticas da pesquisa

Temáticas/Subtemáticas

Trabalhos de História da Matemática apoiado por TIC com uso 2 de software(1.1)

Trabalhos de História da Matemática apoiado por TIC com o uso 1 de outras mídias informáticas(1.2)

Trabalhos de TIC com o uso de software(2.1) 0

Trabalhos de TIC com o uso de outras mídias informáticas(2.2) 1

Trabalhos de História da Matemática(3) 25

Trabalhos de outras áreas de Educação Matemática(4) 88

Total de trabalhos apresentados 117

Fonte: Pesquisa realizada nos anais do VII Encontro Luso-Brasileiro de História da Matemática (Outubro, 2014)

Nesse evento e nessa modalidade de apresentação - comunicação - encontramos dois trabalhos na subtemática 1.1. Apresentaremos esses trabalhos a seguir.

Em Um olhar as curvas de Descartes, construídas por instrumentos, pelo aplicativo geométrico GeoGebra, Eduardo Sebastiani Ferreira traz a construção das Curvas de Descartes, antes feitas por processos mecânicos, que após construção, podese introduzir coordenadas e propor as equações que representavam essas curvas.

Eduardo Sebastiani Ferreira, Otilia T. W. Paques e Rosa Maria Machado, em Conchóides de René Descartes, trazem o histórico dos Conchóides de Nicomedes, considerada uma das curvas resultada de processos mecânicos, como sendo uma possível solução para se resolver um dos três problemas clássicos da geometria grega: a trissecção do ângulo. 
Nesse mesmo evento há outras categorias de apresentação como pôsteres e exposições, muito embora, dentre essas, apenas na de pôsteres obtivemos trabalhos que trouxeram a aliança entre a HM e as TIC. A seguir, podemos visualizar os dados dos pôsteres.

Tabela 2 - Pôsteres apresentados no VII Encontro Luso-Brasileiro de História da Matemática

\begin{tabular}{lc}
\hline Temáticas/Subtemáticas & Quantidade \\
\hline $\begin{array}{l}\text { Trabalhos de História da Matemática apoiado por TIC com uso } \\
\text { de software (1.1) }\end{array}$ & 1 \\
\hline $\begin{array}{c}\text { Trabalhos de História da Matemática apoiado por TIC com o uso } \\
\text { de outras mídias informáticas (1.2) }\end{array}$ & 0 \\
\hline Trabalhos de TIC com o uso de software (2.1) & 0 \\
\hline Trabalhos de TIC com o uso de outras mídias informáticas (2.2) & 12 \\
\hline Trabalhos de História da Matemática (3) & 12 \\
\hline Trabalhos de outras áreas de Educação Matemática (4) & 27 \\
\hline Total de trabalhos apresentados
\end{tabular}

Fonte: Pesquisa realizada nos anais do VII Encontro Luso-Brasileiro de História da Matemática (Outubro, 2014)

Apresentaremos a seguir o trabalho encontrado que, segundo a nossa análise, traz a conjunção entre HM e TIC:

Em A História da Matemática como Ferramenta de ensino e aprendizagem no Ensino Médio: a importância do número Phi, os autores, Hildebrando Almeida de Andrade, Vilmar Gomes da Fonseca André Luiz Souza Silva e José Carlos Gonçalves Gaspar, abordam, através de uma pesquisa qualitativa, como os alunos do ensino médio compreendem a História da Matemática, sobretudo e especificamente, a importância e a história do numero phi, por meio do contato com a digressão histórica apresentada com a exposição utilizando-se o software Geogebra.

b) Levantamento dos dados do VI SIPEM - Seminário Internacional de Pesquisa em Educação Matemática

O evento foi realizado em Pirenópolis, Goiás, Brasil, de 15 a 19 de novembro, no ano de 2015. Teve como o objetivo divulgar as produções brasileiras no âmbito da Educação Matemática, além de promover a interação entre os pesquisadores que têm como objeto de estudo a Educação Matemática. O primeiro SIPEM ocorreu no período de 22 a 25 de novembro do ano de 2000, na cidade de Serra Negra, São Paulo.

Faz-se necessário a apresentação de uma tabela com os dados obtidos do levantamento feito das comunicações apresentadas nesse evento, de acordo com nossa classificação/categorização.

Tabela 3 - Comunicações apresentadas no VI SIPEM 
Giselle Costa de Sousa, Allyson Emanuel Januário da Costa

Investigando a Conjunção entre História da Matemática e Tecnologias de Informação e Comunicação, por meio de um Levantamento Bibliográfico em Eventos Internacionais de Educação Matemática

\begin{tabular}{cc}
\hline Subtemáticas & Quantidade \\
\hline $\begin{array}{c}\text { Trabalhos de História da Matemática apoiado por TIC com uso } \\
\text { de software (1.1) }\end{array}$ & 1 \\
\hline $\begin{array}{c}\text { Trabalhos de História da Matemática apoiado por TIC com o } \\
\text { uso de outras mídias informáticas (1.2) }\end{array}$ & 0 \\
\hline Trabalhos de TIC com o uso de software (2.1) & 17 \\
\hline Trabalhos de TIC com o uso de outras mídias informáticas (2.2) & 5 \\
\hline Trabalhos de História da Matemática (3) & 124 \\
\hline Trabalhos de outras áreas de Educação Matemática (4) & 169 \\
\hline Total de trabalhos apresentados &
\end{tabular}

Fonte: Pesquisa realizada nos anais do VI SIPEM (Novembro, 2015)

Após a apresentação quantitativa dos dados desse evento, como visto, encontramos 1 trabalho que se adequa a subtemática 1.1 que estamos procurando, como podemos observá-lo a seguir:

Em Direções Para Uma Filosofia Geométrica Das Transformações, Adlai Ralph Detoni e José Milton Lopes Pinheiro abordam as diferentes geometrias, por meio da compreensão do cenário histórico e epistemológico em que estão inseridas, tratadas dentro de um ambiente gráfico, com aplicação de um software dinâmico, a fim de entender o surgimento da Geometria das Transformações.

A fim de encontrar a conjunção entre HM e TIC, dos trabalhos catalogados anteriormente, selecionamos os da subtemática 1.1 - Trabalhos de História da Matemática apoiado por TIC com uso de software - para passar por uma análise que os estuda observando aspectos/parâmetros, como: referencial, metodologia, tema histórico, software utilizado (recurso tecnológico), argumentos favoráveis encontrados nos trabalhos e cunho educacional - presença ou não de um produto educacional que segundo a Capes (2016) é o resultado do Trabalho de Conclusão de Curso de um Mestrado Profissional, devendo ser aplicado em sala de aula ou em outro espaço escolar. Ele pode ser uma sequência didática, um jogo, um vídeo ou até mesmo um aplicativo de computador. Ressaltamos que a subtemática supracitada (1.1) foi escolhida, pois consideramos o cerne da conjuntura aqui proposta.

\section{Análise dos Trabalhos}

Os 4 trabalhos encontrados foram analisados, procurando evidenciar a validade da conjunção, bem como, se o uso de TIC (software) pode potencializar a HM, para que possamos identificar como pesquisas na área da Educação Matemática estão tratando esse tema e como essa conjunção está sendo abordada. Assim, apresentamos o quadro a 

por meio de um Levantamento Bibliográfico em Eventos Internacionais de Educação Matemática

seguir. Nele, os Argumentos Favoráveis para conjunção entre HM e TIC serão apresentados no item 4.1.4 sob forma de citações retiradas dos trabalhos encontrados na subtemática 1.1, que façam alusão às partes colocadas no item Analisando os Argumentos Favoráveis deste artigo. Para diferenciar estas das demais citações do referencial deste trabalho (considerando os dados coletados) as destacaremos em itálico e centralizado no texto. Além disso, o referencial dos trabalhos analisados é posto em negrito e caixa alta para diferenciar do nosso. A identificação do Cunho Educacional neste trabalho se dará através da procura de Produtos Educacionais, como destacado no item Analisando o Cunho Educacional.

Consideramos que o quadro posto reflete a conjunção entre HM e TIC, pois podemos comparar, de forma ampla, parâmetros encontrados nos trabalhos. De fato, dos itens colocados (das amostras dos trabalhos dos eventos estudados), nota-se que o autor Eduardo Sebastiani Ferreira foi o que mais contribuiu para a conjunção. Além disso, podemos observar que a modalidade de apresentação é variada, mas que a presença maior foi no evento VII Encontro Luso-Brasileiro de HM.

Quadro 1 - Análise dos trabalhos encontrados na subtemática 1.1

\begin{tabular}{|c|c|c|c|c|c|c|c|c|c|}
\hline EVENTO & MODALIDADE & TITULO & AUTORES & REFERENCIAL & METODOLOGIA & $\begin{array}{c}\text { TEMA } \\
\text { HISTORICO }\end{array}$ & $\begin{array}{c}\text { RECURSO } \\
\text { TECNOLOGICO }\end{array}$ & \begin{tabular}{|c|} 
ARGUMENTOS FAVORAVEIS \\
PARA ALIANÇA
\end{tabular} & $\begin{array}{c}\text { CUNHO } \\
\text { EDUCACIONAL } \\
\end{array}$ \\
\hline $\begin{array}{c}\text { VI } \\
\text { Encontro } \\
\text { Luso- } \\
\text { Brasileiro } \\
\text { de História } \\
\text { da } \\
\text { Matemática }\end{array}$ & Comunicação & $\begin{array}{l}\text { Um olhar as } \\
\text { curvas de } \\
\text { Descartes, } \\
\text { construidas por } \\
\text { instrumentos, } \\
\text { pelo aplicativo } \\
\text { geométrico } \\
\text { Geogebra }\end{array}$ & $\begin{array}{l}\text { Eduardo } \\
\text { Sebastiani } \\
\text { Ferreira }\end{array}$ & $\mathrm{x}$ & $\mathrm{x}$ & $\begin{array}{l}\text { Geometria: } \\
\text { Curvas de } \\
\text { Descartes } \\
\text { (explicito) }\end{array}$ & Software Geogebra & $\begin{array}{l}\text { Utiliza-se o software (Geogebra) } \\
\text { no sentido de dar um novo } \\
\text { significado a um método antigo de } \\
\text { representar a geometria. }\end{array}$ & $\mathrm{x}$ \\
\hline \begin{tabular}{c|} 
VI \\
Encontro \\
Luso- \\
Brasileiro \\
de História \\
da \\
Matemática \\
\end{tabular} & Comunicação & $\begin{array}{l}\text { Conchóides de } \\
\text { René Descartes }\end{array}$ & \begin{tabular}{|c|} 
Eduardo \\
Sebastiani \\
Ferreira, \\
Otilia T. W. \\
Paques e \\
Rosa Maria \\
Machado
\end{tabular} & $\mathrm{X}$ & $\mathrm{X}$ & $\begin{array}{l}\text { Geometria: } \\
\text { Conchóides } \\
\text { de Descartes } \\
\text { (explicito) }\end{array}$ & $\begin{array}{l}\text { Software não } \\
\text { explicito }\end{array}$ & $\begin{array}{l}\text { Utilizam-se aplicativos de } \\
\text { geometria dinâmica com o intuito } \\
\text { de conjecturar, visualizar e } \\
\text { experimentar os resultados obtidos. }\end{array}$ & $\mathrm{x}$ \\
\hline $\begin{array}{c}\text { VI } \\
\text { Encontro } \\
\text { Luso- } \\
\text { Brasileiro } \\
\text { de História } \\
\text { da } \\
\text { Matemática }\end{array}$ & Pôster & $\begin{array}{c}\text { A História da } \\
\text { Matemática } \\
\text { como Ferramenta } \\
\text { de ensino e } \\
\text { aprendizagem no } \\
\text { Ensino Médio: a } \\
\text { importância do } \\
\text { número Phi }\end{array}$ & \begin{tabular}{|c|} 
Hildebrand \\
o Almeida \\
de Andrade, \\
Vilmar \\
Gomes da \\
Fonseca \\
André Luiz \\
Souza Silva \\
e José \\
Carlos \\
Gonçalves \\
Gaspar \\
\end{tabular} & $\begin{array}{c}\text { (MIGUEL \& } \\
\text { MIORIM, 2008): A } \\
\text { História na Educação } \\
\text { Matemática: propostas } \\
\text { e desafios. }\end{array}$ & $\begin{array}{l}\text { Pesquisa } \\
\text { Qualitativa }\end{array}$ & $\begin{array}{l}\text { Geometria: A } \\
\text { importância } \\
\text { do número } \\
\text { Phi } \\
\text { (explicita) }\end{array}$ & $\begin{array}{l}\text { Softaware } \\
\text { Geogebra }\end{array}$ & $\begin{array}{l}\text { Utiliza-se o software como uma } \\
\text { digressão histórica do assunto, ou } \\
\text { seja, como uma ruptura na } \\
\text { construção histórica, de modo a } \\
\text { reforçar e trazer uma correlação } \\
\text { entre a história e as ideias obtidas } \\
\text { nas construções do Geogebra. }\end{array}$ & $\begin{array}{l}\text { Sequência de } \\
\text { Atividades }\end{array}$ \\
\hline VII SIPEM & Comunicação & $\begin{array}{l}\text { Direções Para } \\
\text { Uma Filosofia } \\
\text { Geométrica Das } \\
\text { Transformações }\end{array}$ & $\begin{array}{c}\text { Adlai Ralph } \\
\text { Detoni, José } \\
\text { Milton } \\
\text { Lopes } \\
\text { Pinjleiro }\end{array}$ & $\begin{array}{c}\text { (BACHELARD, } \\
\text { 1968): O novo espirito } \\
\text { cientifico; (PIAGET \& } \\
\text { GARCIA, 1987): } \\
\text { Psicogênese e História } \\
\text { das Ciências. }\end{array}$ & $\mathrm{X}$ & $\begin{array}{c}\text { Geometria: } \\
\text { Geometria } \\
\text { das } \\
\text { Transformaçõ } \\
\text { es (explicito) }\end{array}$ & $\begin{array}{l}\text { Software não } \\
\text { explicito }\end{array}$ & $\begin{array}{l}\text { O software na tentativa de } \\
\text { compreender o caráter dinâmico } \\
\text { aliado às altemativas de } \\
\text { estruturaçōes geométricas. Dentro } \\
\text { da história do tratamento } \\
\text { geométrico, qual mais propicio } \\
\text { para se usar software gráfico. }\end{array}$ & $\mathrm{x}$ \\
\hline
\end{tabular}

Fonte: Pesquisa realizada nos anais do VI SIPEM (Novembro, 2015) e nos anais do VII Encontro LusoBrasileiro de História da Matemática (Outubro, 2014)

Outros aspectos serão apresentados na sequência. 


\section{i) Analisando o Referencial}

Tem-se como parâmetro analisado se os trabalhos encontrados têm referencial em comum, com a finalidade de compreender se as referências utilizadas pelos autores são as mesmas, ou seguem uma mesma tendência, já que são trabalhos sobre a conjunção entre HM e TIC. Pelo quadro, vemos que não foi possível encontrar referencial em comum, porém faz-se necessário que destaquemos os referenciais encontrados e analisemos cada um.

No trabalho A História da Matemática como Ferramenta de ensino $e$ aprendizagem no Ensino Médio: a importância do número Phi foi encontrado como referencial A História na Educação Matemática: propostas e desafios (MIGUEL \& MIORIM, 2008), quando evidenciam Andrade, Silva, Fonseca e Gaspar (2014, p. 133) “As considerações positivas relativas à postura e ao desenvolvimento de conhecimento dos participantes confirmam as indicações de MIGUEL e MIORIM." Vale salientar que esse mesmo referencial é compartilhado no presente trabalho, evidenciando que quando se pesquisa História da Matemática, Miguel e Miorim (2008) são fundamentais para a fundamentação teórica.

Em Direções Para Uma Filosofia Geométrica Das Transformações foram encontrados como referencial: O novo espírito científico (BACHELARD, 1968) e Psicogênese e História das Ciências (PIAGET \& GARCIA, 1987). Nesse trabalho destaca-se o percurso histórico das Geometrias, inclusive sob o viés epistemológico de Bachelard (1884-1962), a saber em:

O filósofo diz que um diferencial para a compreensão epistemológica das novas geometrias é o entendimento da ideia de grupo. Sendo o grupo um grupo de invariantes que se manifestam dada uma transformação, promove-se, epistemologicamente, uma grande guinada na definição do que é (são) geometria (s). (DETONI; PINHEIRO, 2015, p. 6)

Nota-se outro referencial teórico, PIAGET E GARCIA (1987), quando Detoni e Pinheiro (2015) trazem evidencias da construção do conhecimento, assim como quando destacam:

(Piaget e Garcia) argumentam que, por um longo período - que abrange séculos - a noção de transformação em geometria foi constantemente aplicada, no entanto ela ainda não era tematizada em seu significado e possibilidades; muitas concepções abstratas em matemática, hoje tematizadas, foram utilizadas sem as atuais reflexões que nos permitem 
justificar sua utilização bem como direcionar seus sentidos a contextos matemáticos distintos. (DETONI; PINHEIRO, 2015, p. 7)

É interessante compreender que tematizar hoje em dia a noção de transformações em Geometria abre muitas oportunidades, principalmente, no que se refere à utilização dela em diferentes contextos, saindo de aplicações e abstrações e encontrando visões atuais e direcionadas.

ii) Analisando a Metodologia

Após a leitura e análise dos trabalhos, pode-se constatar que estes não apresentam uma metodologia explícita, porém em A História da Matemática como Ferramenta de ensino e aprendizagem no Ensino Médio: a importância do número Phi foi desenvolvida uma pesquisa qualitativa, que inferimos ser uma pesquisa-ação, como destacado em:

A pesquisa-ação educacional é principalmente uma estratégia para o desenvolvimento de professores e pesquisadores de modo que eles possam utilizar suas pesquisas para aprimorar seu ensino e, em decorrência, o aprendizado de seus alunos. (TRIPP, 2005, p. 445)

Destacamos isso porque em uma pesquisa-ação há uma participação e cooperação mais efetiva na pesquisa, salientando a pesquisa-ação educacional em que o professor e pesquisador utilizam-se de pesquisas, nesse caso pesquisa envolvendo História da Matemática, para que a aprendizagem seja mais significativa no aluno, como observaram Andrade, Silva, Fonseca e Gaspar (2014, p. 133) "envolvimento muito superior ao registrado em outros momentos pelo professor regente, também autor deste trabalho.”. Muito embora tenhamos encontrado em apenas um trabalho, a metodologia explicitamente apresentada, faz-se necessário compreender quais os métodos, procedimentos e ferramentas estão sendo mais utilizados ao se pensar em pesquisas que abordem conjunção entre HM e TIC.

\section{iii) Analisando o Tema Histórico e Recurso Tecnológico}

Observamos os Tópicos de História da Matemática mais utilizados, relacionando esse parâmetro com o software (Recurso Tecnológico) mais presente nos trabalhos da subtemática 1.1. Ao fazermos uma análise conjunta desses dois parâmetros encontramos, como resultado, que a Geometria foi o tópico de História da Matemática 
(Tema Histórico) mais presente nos quatro trabalhos encontrados, assim como, no aspecto Recurso Tecnológico, destaca-se o software Geogebra, muito embora em alguns trabalhos, como Conchóides de René Descartes, não houve menção a um software explícito, mas que inferimos pela análise ser um software de matemática dinâmica. Certamente a Geometria ganha espaço quando atrelada aos Recursos Tecnológicos, pois nota-se que o software de matemática dinâmica oportuniza ao aluno uma investigação matemática (PONTE; BROCADO; OLIVEIRA, 2009), com ênfase nos aspectos geométricos pela natureza deste mesmo recurso (por exemplo, o Geogebra começou sendo software de Geometria dinâmica), fazendo com que desenvolva seu espírito pesquisador e isso aliado ao ensino de Geometria será fundamental para a aprendizagem da Matemática, a saber em:

Os softwares de Geometria Dinâmica ganham destaque, uma vez que podem ser um forte aliado na investigação em Geometria. Eles são considerados como uma ferramenta poderosa, tanto quando se pensa em ensino quanto em aprendizagem, podendo despertar o interesse tanto do professor quanto do aluno. (ZULATTO, 2002, p. 6)

A outra análise que segue vem da necessidade de investigar quais (se há) argumentos favoráveis ao uso de HM presente nos trabalhos que se usam a conjunção com TIC.

iv) Analisando os Argumentos Favoráveis

Para esta análise foram separados alguns trechos dos trabalhos que, para nós, trazem essa conjunção. Encontramos em Ferreira (2014, p. 96) que

"Essa transformação geométrica de curvas estáticas em problemas que envolvem movimentos, para resultar em lugares geométricos, torna-se hoje, à luz da geometria dinâmica, um

Mas também em: novo caráter educacional e propiciou novas pesquisas."

Um exemplo da aplicação do critério de construtibilidade cartesiano é a explicação do movimento da conchóide, para isso utilizamos aplicativos de geometria dinâmica para visualizar, experimentar e conjecturar os resultados a serem provados. (FERREIRA; PAQUES; MACHADO, 2014, p. 97)

Com essas citações apresentadas, nota-se que a utilização do recurso tecnológico, no caso um software de matemática dinâmica, foi fundamental para o entendimento do problema histórico, como reforçam muito bem Ponte, Brocardo e 
Oliveira (2009) ao destacarem que o processo de investigação matemática surge em torno de um ou mais problemas, passando por quatro momentos essenciais.

Destacam-se os seguintes argumentos em Andrade, Silva, Fonseca e Gaspar (2014, p. 133):

"Ainda que reconhecidas as potencialidades pedagógicas da história da matemática (MIGUEL \& MIORIM, 2008), acreditamos que professores e licenciandos precisam explorar ainda mais esse grande universo."

Com isso, entende-se, a importância da História da Matemática, tanto para pesquisa, quanto para o ensino da Matemática. De fato, esses argumentos encontrados nos trabalhos classificados na subtemática 1.1 reforçam que a exploração maior desse universo se dá, inclusive, com a utilização de TIC para ensinar HM. Ainda de acordo com Andrade, Silva, Fonseca e Gaspar (2014, p. 133) destaca-se:

"os alunos tiveram contato com uma digressão histórica do número Phi apresentada com o uso de vídeos e de exposições com o software GeoGebra."

E também:

Registramos uma tomada de consciência nos processos de aprendizagem que eram foco nas atividades, principalmente considerando os aspectos históricos que foram atribuídos às situações e aos conhecimentos abordados na sequência de atividade. Em especial, registramos êxito maior nos itens em que foi possível constatar uma correlação direta com as ideias da construção do número Phi apresentadas na digressão histórica. (ANDRADE; SILVA;FONSECA; GASPAR, 2014, p. 133)

Podemos inferir, no que se refere à argumentação favorável quanto à conjunção, ao se visualizar a importância do tratamento histórico nessa situação, pois esse tratamento pôde contribuir na estruturação do entendimento histórico dos estudantes, bem como a importância do apoio do software GeoGebra. Isso contribuiu, positivamente, pois permitiu ao aluno fazer uma relação entre o conhecimento adquirido através da digressão e as sequências de atividades feitas em sala de aula, resultando numa maior conscientização do processo de aprendizagem.

Encontramos argumentos, também, em Detoni e Pinheiro (2015, p. 2):

"O tratamento pedagógico usual se abstém de propostas mais investigativas, bastando-se no aporte de propriedades já conhecidas."

Presente também quando menciona que: 
"perceber o potencial pedagógico que se abre para uma geometria escolar, uma vez que há uma dinâmica de relações mais ampla que a geometria elementar, e que pode ser trabalhada." (p. 5)

Já em Detoni e Pinheiro (2015, p. 10), vemos que:

"a importância de se pesquisar de que maneira modos distintos de geometrizar renovam, especialmente em relação à tradição científica e escolar, objetos e focos, objetivos e possibilidades e esquemas conceituais e métodos."

Com essas citações, conseguimos identificar que é importante destacar que entender as novas formas de fazer Geometria podem reforçar o uso de softwares, em especial os de matemática dinâmica, pois eles podem trazer um novo olhar, não só no que se refere à concepção epistemológica da Geometria, mas também à construção. Infere-se também que o percurso histórico da Geometria vem a destacar que novas geometrias são, também, novas oportunidades de geometrizar, desenvolvendo valores pedagógicos importantes tanto para o educador matemático, como para o pesquisador em Educação Matemática, pois renovar - a conjunção entre HM e TIC traz essa renovação - é trazer novos (e melhores) direcionamentos para a Geometria.

Ainda no trabalho Direções Para Uma Filosofia Geométrica Das Transformações, Detoni e Pinheiro (2015, p. 7) destacam:

"Levada para o campo do pedagógico, essa questão se põe abrindo diferenças entre as práticas mais tradicionais do ensino, quando seguimos uma geometria, e uma prática que vai propor geometrizar-se de certo modo possível."

Detoni e Pinheiro (2015, p. 9) mostram ainda que:

"A exploração dessas potencialidades junto a um software gráfico é uma aproximação que entendemos ser pertinente investigar."

E na página seguinte:

Ainda como apontamento de direção para seguimento de nossa pesquisa, uma intuição de que esse dinamismo geométrico tem afinidade com as possibilidades de tratamento dinâmico de objetos geométricos quando estamos juntos a um software gráfico aberto. (DETONI, PINHEIRO, 2015, p. 10)

Com essas citações, conseguimos compreender que com um pensamento sobretudo ligado à prática de ensino pode-se visualizar dentro da história do tratamento geométrico, qual mais propicio para se usar software gráfico.

Outro aspecto a ser considerado foi a observação se os trabalhos têm um direcionamento educacional. Para tanto, tal parâmetro é estudado no item que segue. 
v) Analisando o Cunho Educacional

Dentro dessa análise foi procurado, por nós, Produtos Educacionais, do tipo dos resultados de dissertações de Mestrado Profissional em Ensino, cujo:

O trabalho final do Mestrado Profissional em Ensino deve ser uma pesquisa aplicada, descrevendo o desenvolvimento de processos ou produtos de natureza educacional, visando à melhoria do ensino na área específica, sugerindo-se fortemente que, em forma e conteúdo, este trabalho se constitua em material que possa ser utilizado por outros profissionais. (OSTERMANN, REZENDE, 2009, p. 70)

Contudo, buscamos algo do tipo colocado, mas não necessariamente fruto de uma dissertação profissional. Após o levantamento por esses Produtos não foram encontrados produtos, de forma explícita.

Não obstante, encontramos no trabalho A História da Matemática como Ferramenta de ensino e aprendizagem no Ensino Médio: a importância do número Phi um indício de Produto Educacional, no trecho que diz que há uma "sequencia de atividades que admitia o contato com conteúdos matemáticos de Sequencia e Geometria (Teorema de Pitágoras).”(ANDRADE; SILVA; FONSECA; GASPAR, 2014, p. 133), é possível entender a relação da sequência de atividade com o Produto Educacional, pois segundo Moreira e Nadir (2009) o produto educacional pode vir em forma de texto sobre uma sequência didática, ou $\mathrm{CD}$ e até mesmo aplicativos, mas ainda assim para entender essa relação entre a sequência de atividade, trazida pelo autor do trabalho da subtemática 1.1, e o produto educacional, faz-se necessário que entendamos Sequência Didática, que se define:

\begin{abstract}
A sequência didática é um conjunto de atividades ligadas entre si, planejadas para ensinar um conteúdo, etapa por etapa, organizadas de acordo com os objetivos que o professor quer alcançar para aprendizagem de seus alunos e envolvendo atividades de avaliação que pode levar dias, semanas ou durante o ano. É uma maneira de encaixar os conteúdos a um tema e por sua vez a outro tornando o conhecimento lógico ao trabalho pedagógico desenvolvido. (PERETTI; TONIN DA COSTA, 2013, p. 6)
\end{abstract}

Nota-se que as sequências de atividades desenvolvidas nesse trabalho podem se desdobrar em um Produto, reforçando a conjunção entre HM e TIC, mas de uma forma mais prática. O cunho educacional trazido por esses trabalhos podem se resumir em: ressaltar, à luz da geometria dinâmica, o novo caráter educacional com as transformações geométricas (presente no trabalho Um olhar as curvas de Descartes, construídas por instrumentos, pelo aplicativo geométrico GeoGebra), destacar a 
importância pedagógica da História Matemática, além de um registro de uma tomada de consciência nos processos de aprendizagem (trabalho A História da Matemática como Ferramenta de ensino e aprendizagem no Ensino Médio: a importância do número Phi) e destacar a importância de se tratar o percurso histórico da Geometria, para entender a necessidade de uma reestruturação curricular, sobretudo um tratamento pedagógico mais investigativo (trabalho Direções Para Uma Filosofia Geométrica Das Transformações).

\section{Considerações Finais}

Tendo em vista um levantamento preliminar por trabalhos em eventos da área, no âmbito nacional, assim como uma investigação, em trabalhos acadêmicos que apontou para a escassez da utilização de softwares educacionais na resolução de problemas históricos e ao mesmo tempo assinalou a relevância do uso da História da Matemática e das TIC isoladamente, procurou-se continuar com a busca por trabalhos, no âmbito internacional, a fim de encontrar indícios do uso de ambas as tendências em Educação Matemática, tendo sempre em vista o modo como uma pode potencializar a outra.

Ainda que sejam poucos trabalhos encontrados que trouxeram a conjunção entre HM e TIC, faz-se necessário salientar as possibilidades apontadas por estes de eficiência do uso de ambas.

O levantamento de pesquisa possibilitou verificar que as investigações voltadas para a conjunção entre a HM e TIC possuem duas abordagens: a ampliação do uso dessas tendências pelo professor de Matemática da Educação Básica e despertar nos pesquisadores, em Educação Matemática, a importância do tema. Observamos a presença maior da conjunção no âmbito de pesquisa em Matemática, ou em EM e em HM. Nestes casos, pode-se notar que os resultados positivos de uso destas tendências conjuntamente ocorrem com base em pesquisas qualitativas em sua maioria que se apoiam em referências de HM e TIC, ainda isoladamente, com maior ênfase no tema Geometria e recorrência do uso de software Geogebra. Contudo, aludimos que há outras possibilidades especificamente via IM que também usam HM e TIC. 


\section{Referências}

ANDRADE, H. A. de; FONSECA, V. G. da; SILVA, A. L. S.; GASPAR, J. G. A História da Matemática como Ferramenta de ensino e aprendizagem no Ensino Médio: a importância do número Phi. In: ENCONTRO LUSO-BRASILEIRO DE HISTÓRIA DA MATEMÁTICA, 7., 2014, Óbidos. Resumos... Óbidos: Sociedade Portuguesa de Matemática, 2014. p. 133.

BORBA, Marcelo de Carvalho; PENTEADO, Miriam Godoy. Informática e Educação Matemática. 3 ed. Belo Horizonte: Autêntica, 2007.

CAPES. Orientações para APCN. Coordenação de Aperfeiçoamento de Pessoal de Nível Superior. Brasília: Ministério da Educação, 2016.

DETONI, A. R.; PINHEIRO, J. M.L. Direções Para Uma Filosofia Geométrica Das Transformações. In: SEMINÁRIO INTERNACIONAL DE PESQUISA EM EDUCAÇÃO MATEMÁTICA, 6., 2015, Pirenópolis. Anais... Brasília: SBEM, 2015. p. $1-11$.

ENCONTRO Luso-Brasileiro de História da Matemática. 7. Óbidos. 2014. CD ROOM.

FERREIRA, E. S; PAQUES, O. T. W; MACHADO, R.M. Conchóides de René Descartes. In: ENCONTRO LUSO-BRASILEIRO DE HISTÓRIA DA MATEMÁTICA, 7., 2014, Óbidos. Resumos... Óbidos: Sociedade Portuguesa de Matemática, 2014. p. 97.

FERREIRA, E. S. Um olhar as curvas de Descartes, construídas por instrumentos, pelo aplicativo geométrico GeoGebra. In: ENCONTRO LUSO-BRASILEIRO DE HISTÓRIA DA MATEMÁTICA, 7., 2014, Óbidos. Resumos... Óbidos: Sociedade Portuguesa de Matemática, 2014. p. 96.

GODOY, Arilda Schmidt. Pesquisa Qualitativa Tipos Fundamentais. Revista de Administração de Empresas, São Paulo, v. 35, n.3, p. 20-29 Mai./Jun. 1995.

LIMA, Telma Cristiane Sasso de; MIOTO, Regina Célia Tamaso. Procedimentos metodológicos na construção do conhecimento científico: a pesquisa bibliográfica. Revista Katál, Florianópolis, v. 10 n. esp. p. 37-45, 2007.

MIGUEL, Antonio; MIORIM, Maria Ângela. História na Educação Matemática: propostas e desafios. - 1 ed., 2 reimp. - Belo Horizonte: Autêntica, 2008.

MOREIRA, M. A.; NARDI, R. Mestrado Profissional na área de Ensino de Ciências e Matemáticas: alguns esclarecimentos. R. B. E. C. T., v. 2, n. 3, set./dez. 2009.

NEVES, Jose Luis. Pesquisa Qualitativa - características, usos e possibilidades. São Paulo: Caderno de Pesquisa em Administração, 1996. 
OSTERMANN, F.; REZENDE, F. Projetos de desenvolvimento e de pesquisa na área de ensino de ciências e matemática: uma reflexão sobre os mestrados profissionais. Cad. Bras. Ens. Fís., v. 26, n. 1: p. 66-80, abr. 2009.

PERETTI, Lisiane; TONIN DA COSTA, Gisele Maria. Sequência Didática na Matemática. Revista de Educação do IDEAU, v. 8, n. 17, jan./jun. 2013.

PONTE, João Pedro da; BROCARDO, Joana; OLIVEIRA, Hélia. Investigações Matemáticas na Sala de Aula. - 2. Ed. - Belo Horizonte: Autêntica Editora, 2009.

SEMINÁRIO Internacional de Pesquisa em Educação Matemática. 6. Pirenópolis. 2015. Disponível em: 〈http://www.sbembrasil.org.br/visipem/anais/story>. Acesso em: 25 de Junho de 2016.

TRIPP, David. Pesquisa-ação: uma introdução metodológica. Educação e Pesquisa, São Paulo, v. 31, n. 3, p. 443-466, set./dez. 2005.

ZORZAN, Adriana Salete Loss. Ensino-aprendizagem: algumas tendências na educação matemática. R. Ciências Humanas, v. 8, n. 10, p. 77-93, Jun. 2007.

ZULATTO, Rúbia Barcelos Amaral. Professores de matemática que utilizam softwares de geometria dinâmica: suas características e perspectivas. 2002. 184f. Dissertação (Mestrado em Educação Matemática) - Universidade Estadual Paulista, Rio Claros, 2002. 